\title{
Achados Imaginológicos que Indicam Proximidade das Raízes de Terceiros Molares Inferiores com o Canal Mandibular: Revisão de Literatura
} Imaginological Findings that Indicate Proximity of the Roots of Lower Molar Third Parties with the Mandibular Channel: Literature Review

Hallazgos Imaginológicos que Indican la Proximidad de Raíces de Terceros Molares Inferiores con el Canal Mandibular: Revisión de la Literatura

Karolina Braga CostA Graduanda em Odontologia da Faculdade de Odontologia da Universidade Federal de Mato Grosso do Sul (FaodoUFMS) e membro da Liga Acadêmica de Cirurgia e Traumatologia Buco-maxilo-facial (LACTBMF) da Faodo-UFMS Yuri NEJAIM

Docente da Graduação em Odontologia da Faodo-UFMS, Radiologia Oral https://orcid.org/0000-0002-0442-2601

Ellen Cristina GAETTI-JARDIM

Docente da Graduação em Odontologia da Faodo-UFMS, Preceptora da Residência em Cirurgia e Traumatologia Bucomaxilofacial da Faodo-UFMS e da LACTBMF https://orcid.org/0000-0003-2471-465X

\section{Resumo}

A radiografia panorâmica (RP) é o exame de imagem mais utilizado para avaliar a relação entre as raízes do terceiro molar inferior (TMI) com o canal mandibular (CM), no entanto a tomografia computadorizada de feixe cônico (TCFC) também pode ser indicada com a mesma finalidade, tendo a vantagem de permitir uma análise tridimensional da região. O objetivo deste estudo foi realizar um levantamento bibliográfico sobre os sinais radiográficos que indicam relação de proximidade entre a raiz dentária do TMI com o CM na RP e TCFC. Buscas foram feitas no PubMed, Medline e Lilacs empregando os seguintes descritores: Radiography Panoramic, Mandibular Nerve, Molar Third, Cone-Beam Computed Tomography. Foi realizada a leitura completa dos textos compatíveis com os objetivos, excluindo àqueles em que o $\mathrm{CM}$ não apresentava íntimo contato com as raízes do TMI nos exames de imagens, relatos de casos clínicos e artigos que utilizaram radiográficas periapicais para observar tal relação. Os sinais radiográficos mais relevantes para avaliar a relação raiz/canal mandibular foram: interrupção da linha branca do canal, escurecimento da raiz, desvio/estreitamento do canal, bem como sinais tomográficos incluindo ausência de corticalização, posição lingual do nervo e formato anatômico do canal (lágrima e haltere), como preditores de maior risco para lesão nervosa. Conclui-se que o exame de eleição para avaliar a proximidade do TMl com o CM continua sendo a RP, tendo em vista que ela apresenta sinais confiáveis, menor dose de radiação e custo se comparado à TCFC.

Descritores: Radiografia panorâmica; Tomografia Computadorizada de Feixe Cônico; Terceiro Molar; Nervo Mandibular.

\section{Abstract}

Panoramic radiography $(\mathrm{RP})$ is the most widely used imaging test to evaluate the relationship between the roots of the lower third molar (LTM) and the mandibular canal (MC), however the cone beam computed tomography (CBCT) can also be indicated for the same purpose, due to its advantage of allowing a three-dimensional analysis of the region. The aim of this study was to carry out a bibliographic survey on radiographic signs that indicate a close relationship between the dental root of the LTM with the MC on PR and CBCT. Searches were made on PubMed, Medline and Lilacs using the following descriptors: Panoramic radiography, Mandibular Nerve, Third molar, Cone-Beam Computed Tomography. A complete reading of the texts compatible with the objectives was performed, excluding those in which the MC did not have intimate contact with the roots of the LTM in the image exams alongside clinical case reports and articles that used periapical radiographs to observe such a relationship. The most relevant radiographic signs to assess the root and mandibular canal relationship were: interruption of the white line of the canal, root darkening, deviation / narrowing of the canal, as well as signs observed over tomography which include absence of corticalization, lingual nerve position and anatomical shape of the canal (tear-drop and dumbbell), as predictors of higher risk for nerve damage. It is concluded that the exam of choice to evaluate the proximity of the LTM with the MC remains the PR, considering that it presents reliable signs, lower radiation dose and cost when compared to the CBCT.

Descriptors: Radiography Panoramic; Cone-Beam Computed Tomography; Molar Third; Mandibular Nerve.

Resumen

La radiografía panorámica $(\mathrm{RP})$ es la prueba de imagen más utilizada para evaluar la relación entre las raíces del tercer molar inferior (TMI) con el canal mandibular (CM), sin embargo, la tomografía computarizada de haz cónico (TCHC) también puede estar indicada con la misma propósito, teniendo la ventaja de permitir un análisis tridimensional de la región. El objetivo de este estudio fue realizar un relevamiento bibliográfico sobre los signos radiográficos que indican una estrecha relación entre la raíz dentaria del TMI con el CM en el RP y TCHC. Las búsquedas se realizaron en PubMed, Medline y Lilacs utilizando los siguientes descriptores: radiografía panorámica, nervio mandibular, tercer molar, tomografía computarizada de haz cónico. Se realizó una lectura completa de los textos compatibles con los objetivos, excluyendo aquellos en los que el CM no tuvo contacto íntimo con las raíces del TMl en los exámenes de imagen, reportes de casos clínicos y artículos que utilizaron radiografías periapicales para observar tal relación. Las señales radiográficas más relevantes para evaluar la relación raíz / conducto mandibular fueron: interrupción de la línea blanca del conducto, oscurecimiento de la raíz, desviación / estrechamiento del conducto, así como signos tomográficos que incluyen ausencia de corticalización, posición del nervio lingual y forma anatómica del canal (desgarro y mancuerna), como predictores de mayor riesgo de daño nervioso. Se concluye que el examen de elección para valorar la proximidad del TMI con el CM sigue siendo el RP, considerando que presenta signos fiables, menor dosis de radiación y coste con respecto al TCHC.

Descriptores: Radiografía Panorámica; Tomografía Computarizada de Haz Cónico; Tercer Molar; Nervio Mandibular.

INTRODUÇÃO

O terceiro molar inferior (TMI), em comparação com outros dentes da dentição permanente, possui uma grande variação no padrão anatômico. Estes diferem com relação a posição de implantação na mandíbula, tamanho, tempo de desenvolvimento e erupção ${ }^{1}$.
A falta de espaço evolutiva no arco mandibular para irrupção desse elemento e sua posição mais posterior quando erupcionado, que dificulta a higienização adequada, podem desencadear 0 surgimento de patologias comprometendo até mesmo a integridade do dente adjacente ${ }^{2,3}$. 
Alguns estudos mostram que existe alta prevalência de patologias como cárie, doença periodontal, pericoronarite, infecção odontogênica, impacção, reabsorção radicular, cistos, tumores, associadas ao TMI indicando necessidade de exodontia desses dentes ${ }^{4-6}$. Nesses casos, a solicitação de exames complementares de imagem, como radiografia panorâmica (RP) e tomografia computadorizada de feixe cônico (TCFC) é fundamental para realização do diagnóstico, planejamento cirúrgico e acompanhamento do quadro clínico do paciente.

A RP é um exame de imagem bidimensional apontado como primeiro exame de escolha para abordagem diagnóstica das lesões mandibulares, bem como avaliação da relação entre as raízes do $\mathrm{TMI}$ com o $\mathrm{CM}$, pois fornece uma visão geral da mandíbula e de todos os dentes na mesma imagem ${ }^{5,7,8}$. A proximidade visual da raiz do TMl e o canal em uma imagem panorâmica é um indicativo de maior risco para lesão do nervo alveolar inferior no procedimento cirúrgico. Como a maioria dos dentistas utiliza esse método de imagem complementar para avaliação pré-operatória, a precisão dessa modalidade de imagem precisa ser examinada de perto ${ }^{9}$.

Alguns sinais de alto valor preditivo para indicar tal proximidade ou íntimo contato entre as estruturas são identificados na $\mathrm{RP}^{10}$. Dentre eles, destaca-se características relacionadas à raiz (escurecimento da raiz, deflexão da raiz, estreitamento da raiz, ápice radicular escuro e bífido) e sinais associados ao canal (interrupção da linha branca do canal, desvio e/ou estreitamento do canal) ${ }^{11}$.

A TCFC fornece uma visão préoperatória tridimensional, precisa e detalhada da anatomia local ${ }^{12}$. Os benefícios incluem visão de múltiplos planos (axial, coronal e sagital), além de permitir uma reconstrução 3D da região ${ }^{13}$. Todavia, está associada a desvantagens como custos e níveis de exposição à radiação mais elevados se comprados com a RP ${ }^{14}$. Grunheid et al. ${ }^{15}$ relata que a dose de radiação emitida na TCFC equivale de 3.22 a 6.24 radiografias panorâmicas. Por conta disso, a incidência tomográfica é indicada especialmente quando a radiografia não é suficientemente confiável para auxiliar o profissional quanto ao risco real de lesão nervosa durante o procedimento clínico, uma vez que, por ser um exame bidimensional as estruturas observadas aparecem sobrepostas na imagem ${ }^{16}$.

A escolha clínica de qual exame de imagem deve ser utilizado baseia-se no princípio ALADA (As Low as Diagnostically Acceptable being Indication-oriented and Patient-specific) de proteção radiológica, ou seja, a dose de radiação deve ser tão baixa quanto aceitável para o diagnóstico ${ }^{17}$. Dessa maneira, é importante fazer uma revisão na literatura sobre os sinais radiográficos panorâmicos que são mais confiáveis para estabelecer a relação do terceiro molar inferior com o canal mandibular para evitar a realização de exames de TCFC desnecessariamente.

O objetivo deste estudo foi realizar um levantamento bibliográfico sobre os sinais radiográficos que indicam uma relação de proximidade entre a raiz dentária de terceiros molares inferiores e o canal mandibular em radiografias panorâmicas e tomografias computadorizadas de feixe cônico.

MATERIAL E MÉTODO

A revisão de literatura foi estruturada a parti de buscas em bases de dados incluindo o PubMed, Medline e Lilacs. Os seguintes descritores foram empregados: Radiography Panoramic (radiografia panorâmica), Mandibular Nerve (nervo mandibular), Molar Third (terceiro molar), Cone-Beam Computed Tomography (tomografia computadorizada de feixe cônico). Cada um desses termos foi combinado utilizando os operadores booleanos E/OU (AND/OR).

Com auxílio da busca eletrônica foram selecionados os artigos publicados entre os anos de 2010 a 2020. Realizaram-se buscas manuais adicionais por artigos clássicos relevantes para o estudo. $O$ processo de seleção foi realizado com auxílio do gerenciador de referências Mendeley, sendo que o aplicativo removeu automaticamente os artigos duplicados identificados no levantamento da literatura.

Dois revisores selecionaram os artigos pela leitura de títulos e resumos. Após esse processo de seleção, os textos completos compatíveis com os objetivos do trabalho foram lidos, excluindo os estudos em que o canal mandibular não apresentava íntimo contato com as raízes do terceiro molar inferior nos exames de imagens, relatos de casos clínicos e artigos que utilizaram radiográficas periapicais para observar tal relação.

REVISÃO DA LITERATURA

O manejo dos terceiros molares é um tema controverso no que diz respeito a indicação de intervenção cirúrgica, principalmente em dentes assintomáticos sem patologias aparente ${ }^{18}$. Todavia, é certo que 0 tratamento envolve o diagnóstico correto com base nos exames clínicos, radiográficos, planejamento com ênfase na modificação dos 
fatores de risco que podem estar presentes, técnicas cirúrgicas adequadas para minimizar as chances de complicações possibilitando um procedimento com resultados satisfatórios para o paciente ${ }^{19}$.

A solicitação de exames de imagem previamente a exodontia de um TMl é viável na prática clínica porque direciona o profissional na escolha do plano de tratamento. Idealmente, a imagem deve exibir o dente em questão na sua totalidade, dente vizinho e o osso circundante, além das estruturas anatômicas relacionadas $^{20,21}$.

A remoção desses elementos é por vezes dificultada. Existem fatores relacionados a morfologia, número de raízes, espessura do ligamento periodontal, densidade óssea mandibular, presença ou ausência do folículo dentário, proximidade de dentes adjacentes e/ou de estruturas nobres como nervo alveolar inferior (NAI) e nervo lingual que podem trazer complicações ao procedimento ${ }^{6,19,22}$. Por conta disso, os exames de imagens tornam-se ferramentas valiosas para elucidar riscos e o prognóstico de cada caso.

Em uma imagem radiográfica da região do terceiro molar apenas o curso do NAI que se aloja no CM pode ser estimado, sendo que a relação anatômica entre essas estruturas é o fator de risco mais evidente para lesão nervosa $^{23,24}$. Por essa razão, a avaliação radiológica pré-operatória deve ser realizada na tentativa de identificar tal proximidade, pois a taxa do comprometimento sensorial resultante de uma disfunção transitória do NAI ocorre em 0,6 a $5,5 \%$ dos casos, e a disfunção permanente em 0,1 a $0,9 \%$ ocasionando redução ou ausência total de sensibilidade na região de lábio inferior e mento ${ }^{25}$.

- Sinais preditores na radiografia panorâmica que indicam proximidade do terceiro molar inferior com o canal mandibular

Rood e Shehab ${ }^{26}$ categorizaram sete sinais radiográficos (Tabela 1 ), que sugerem a proximidade do $\mathrm{CM}$ com as raízes do TMI. O estudo evidenciou que o desvio do canal, escurecimento da raiz e interrupção da linha branca do canal são achados significativos para lesão do NAI. O desvio do canal mandibular mostrou ser o sinal mais confiável para indicar contato do TMI com o CM. O escurecimento da raiz foi considerado o segundo sinal mais confiável, assumindo a terceira posição o sinal de interrupção da linha branca do canal. O estreitamento da raiz, deflexão da raiz, estreitamento do canal, ápice radicular escuro e bífido, não se mostraram confiáveis indicadores de lesão nervosa pós-operatória.
Tabela 1. Sinais radiográficos da íntima relação do TMl com o $\mathrm{CM}^{26}$

\begin{tabular}{l|l}
\hline Sinais radiográficos & Descrição \\
\hline Escurecimento da raiz & $\begin{array}{l}\text { A imagem aparece mais escura por conta } \\
\text { da perda de densidade na região em que } \\
\text { raiz se encontra dentro do CM }\end{array}$ \\
\hline Raízes defletidas & $\begin{array}{l}\text { Desvio abrupto da raiz quando ela atinge } \\
\text { O CM }\end{array}$ \\
\hline Estreitamento da raiz & $\begin{array}{l}\text { O estreitamento da raiz próximo ao local } \\
\text { que o canal o atravessa, sugere que o } \\
\text { maior diâmetro da raiz foi envolvido pelo } \\
\text { canal, ou que há sulcos profundos ou } \\
\text { perfuração da raiz }\end{array}$ \\
\hline Raiz escura e bífida & $\begin{array}{l}\text { Quando o CM cruza o ápice do dente } \\
\text { aparece uma sombra dando a impressão } \\
\text { de uma raiz bífida }\end{array}$ \\
\hline \multirow{2}{*}{ Interrupção da linha branca do canal } & $\begin{array}{l}\text { São as duas linhas radiopacas que } \\
\text { demarcam a borda "superior e inferior" } \\
\text { do CM. Essas linhas aparecem em uma } \\
\text { radiografia por conta da alta densidade } \\
\text { óssea da estrutura e quando desaparece } \\
\text { da imagem ela é considerada } \\
\text { interrompida }\end{array}$ \\
\hline Desvio do canal alveolar inferior & $\begin{array}{l}\text { Ocorre quando o canal cruza o terceiro } \\
\text { molar inferior e muda sua direção }\end{array}$ \\
\hline Estreitamento do canal alveolar inferior & $\begin{array}{l}\text { Ocorre quando há uma redução do } \\
\text { diâmetro canal ao encontrar a raiz do } \\
\text { TMI }\end{array}$ \\
\hline
\end{tabular}

Utilizando os sinais radiográficos descritos por Rood e Sheehab, Huang et al. ${ }^{9}$, utilizaram imagens panorâmicas para prever o comprometimento sensorial pós-cirúrgico à extração de terceiros molares inferiores impactados. Estatisticamente a interrupção da linha radiopaca, desvio do canal e estreitamento do canal foram associados a lesão do NAI. Nesse estudo apenas os fatores relacionados ao canal mandibular se mostraram significativos para avaliação pré-cirúrgica do risco de parestesia.

Estudos apontam que o escurecimento das raízes e os preditores relacionados ao canal (interrupção das linhas brancas, desvio e estreitamento) são sinais radiográficos promissores ao risco de lesão do NAl, pois indicam associação do dente com o $\mathrm{CM}^{27-29}$. Esses sinais identificados na RP são correlacionados à ausência de corticalização entre o TMI e o NAI ${ }^{12}$, no entanto, é impossível garantir que na ausência deles não exista contato próximo ${ }^{11}$.

Uma revisão sistemática e meta-análise realizada por Atieh ${ }^{21}$, incluiu os três marcadores radiográficos - escurecimento da raiz, interrupção das bordas radiopacas do canal mandibular e desvio do canal mandibular descritos como mais significativos para a previsão de uma relação estreita entre o NAl e $\mathrm{TM}^{26}$. Os resultados mostraram que a RP possui uma razoável acurácia diagnóstica para avaliação pré-operatória da relação entre os terceiros molares e o $\mathrm{CM}$, no entanto ela é mais confiável para excluir a relação estreita entre a raiz e o nervo na ausência desses marcadores do que para confirmar a presença de uma relação verdadeira na presença desses achados, devido à natureza bidimensional da imagem ${ }^{21}$.

Em resumo, os principais sinais panorâmicos identificados em diferentes 
estudos incluem: interrupção da linha branca do canal, escurecimento da raiz, desvio e estreitamento do canal ${ }^{16,30-32}$. A interrupção das bordas radiopacas é o sinal mais prevalente nos casos reais de exposição ao NAI, seguido pelo escurecimento da raiz, por isso são considerados eficazes na determinação de risco de lesão, tanto como achados isolados, quanto associados a outros preditores positivos ${ }^{33}$.

$$
\text { - Sinais preditores na Tomografia }
$$
Computadorizada que indicam proximidade do terceiro molar inferior com o canal mandibular

Os fatores preditivos apontados pela TCFC estão relacionados a forma anatômica do canal, posição do NAl e estado da cortical óssea $^{34}$. Se houver estreita relação entre 0 dente o nervo, a lesão pode ocorrer durante o processo cirúrgico devido as forças compressivas geradas pelo procedimento ${ }^{35,36}$.

Num estudo retrospectivo, Eyrich et al. ${ }^{37}$ aponta sinais na TCFC como estreitamento do $\mathrm{CM}$, ausência de osso esponjoso entre o nervo e o dente, ou seja, contato direto entre as duas estruturas, posição lingual do NAl e/ou entre as raízes do TMI como preditores de alto risco. Além disso, a presença de múltiplas raízes associadas a perfuração do $\mathrm{CM}$ também é levada em consideração ${ }^{35}$, visto que, a imagem de TC parece fornecer informações precisas sobre a morfologia da raiz do terceiro molar e sua relação com o NAI, permitindo identificar os casos em que o nervo está exposto e os casos em que uma impressão dele está presente na superfície da raiz ${ }^{38}$.

Um outro estudo envolvendo a TCFC categorizou a forma anatômica do canal pela análise de cortes tomográficos em 3 grupos: formato redondo/oval, forma de lágrima e haltere. A imagem dos pacientes da amostra que apresentaram alguma alteração na sensibilidade exibia ausência de cortical óssea em $100 \%$ dos casos, apresentando anatomia do canal no padrão redondo/oval ou em forma de haltere. As análises estatísticas concluíram que a ausência de corticalização entre o NAl e o TMI nas imagens de TC é um preditor confiável de lesão nervosa, sendo que, pacientes com canais em formato de haltere apresentam alto risco de alteração da sensibilidade no pósoperatório $^{34}$. Esse tipo de anatomia não é rara, sendo importante 0 conhecimento dessas variáveis no pré-operatório para realizar o planejamento cirúrgico ${ }^{39-41}$.

Ao analisar estudos que investigam a taxa de lesão do NAl de acordo com as características da TCFC é concordante entre os autores que os casos onde o canal mandibular é localizado lingualmente apresentando formato de haltere são mais vulneráveis a lesões ${ }^{42,40,24}$, bem como, a ausência de corticalização ${ }^{43,34}$. Porquanto, Tachinami et al. inferiu que casos apresentando preditores como ausência de corticalização, NAI localizado lingualmente e o $\mathrm{CM}$ em forma de haltere, ao mesmo tempo, possui chances mais significativas para lesão do NAI ${ }^{44}$

- Associação dos sinais panorâmicos aos de Tomografia computadorizada

Alguns sinais específicos na RP quando comparados com os achados na TCFC são significativamente mais propensos a indicar relação de proximidade das raízes do TMI com o CM, incluindo a interrupção da linha branca e escurecimento das raízes ${ }^{27}$. Esses sinais com base nos dois exames de imagem são preditivos para 0 íntimo contato $^{7}$, sendo substancialmente associados a alterações sensoriais no pós operatório ${ }^{39}$.

Em estudo que relaciona os sinais encontrados em RP com a TCFC evidenciou que o escurecimento da raiz ocorre devido à perda de substância calcificada das placas corticais vestibular e / ou lingual. A descontinuidade da linha radiopaca do canal através da raiz indicou em todos os casos contato da raiz com o NAI, pois há perda da estrutura cortical do canal e confirma potencial lesão ao nervo durante a extração. $O$ desvio do canal por sua vez, na tomografia revela íntimo contato do nervo com as estruturas ao passar entre as raízes devido à restrição de espaço na mandíbula. Sendo assim, fica evidente que os 3 sinais confirmados por TCFC são preditivos de um risco aumentado para lesão do $\mathrm{NAl}^{36}$.

Nas reconstruções coronais de TCFC, o contato direto entre o TMl e o $\mathrm{CM}$, juntamente com o estreitamento e/ou fenestração do $\mathrm{CM}$, foi observado com mais frequência quando 2 ou mais sinais panorâmicos de alto risco foram vistos simultaneamente nas imagens de $\mathrm{RP}^{45}$. O desvio do canal e a interrupção das linhas brancas foram associados à perda da corticalização, indicando um contato direto entre as raízes e o canal mandibular ${ }^{46}$.

Os sinais panorâmicos já mencionados, mostram possuir um alto valor preditivo negativo indicando que na ausência deles, a chance de ocorrer lesão nervosa devido à proximidade da raiz do TMI com o CM é mínima. Por outro lado, o valor preditivo positivo é baixo, ou seja, a presença de tais achados não indica necessariamente risco de lesão do $\mathrm{NAl}^{16}$. O mesmo ocorre quando avaliamos um achado de interesse particular na TCFC, a integridade do CM. Existe uma limitação da medida binária para avaliar a probabilidade de exposição do 
nervo, isto significa que na imagem tomográfica este sinal possui alta sensibilidade e valor preditivo negativo, mas baixa especificidade e valor preditivo positivo ${ }^{14}$.

DISCUSSÃO

Os sinais radiográficos apresentados têm sido considerados marcadores padrão para identificar a probabilidade de lesão do NAl e são relatados como cruciais na avaliação de risco pré-operatório ${ }^{21}$. A concordância de quais sinais são mais significativos entre os autores não é unanime, sendo que essas discrepâncias podem ser geradas pelas diferenças na metodologia dos estudos, tamanho da amostra, método para avaliação da lesão nervosa e interpretação subjetiva dos observadores que inferi viés aos estudos.

Alguns autores consideram que os sinais radiográficos de Rood e Sheehab estão frequentemente associados à ausência de corticalização entre a raiz do TMI e o CM, portanto, o exame de TCFC antes da extração cirúrgica é indicado para que as complicações neurológicas pós-operatórias possam ser minimizadas ${ }^{12,37,47}$. Contudo, outros pesquisadores entendem que o uso de imagens tridimensionais não parece reduzir o número de lesões nervosas, nem afeta seu prognóstico quando comparadas as imagens bidimensionais $^{28,48-51}$.

Muito se questiona a acurácia da imagem panorâmica para prever a possibilidade de uma lesão nervosa durante a exodontia do TMI. Não é apropriado o seu uso para descartar a lesão do NAI no pós-operatório porque o seu valor preditivo positivo avaliando os sete sinais é baixo. No entanto, o seu valor agregado para determinar a presença de desvio do canal, interrupção da linha branca do canal e escurecimento da raiz pode ser considerado suficiente para julgar 0 risco de parestesia ${ }^{11}$. Estatisticamente a RP pode perder cerca de um terço dos casos de exposição, mas um diagnóstico panorâmico positivo tem maior probabilidade de ser uma exposição real e deve ser levado a sério ${ }^{30}$.

As TCFC possibilitaram um melhor entendimento das relações anatômicas dos TMI, isso traz como vantagem a possibilidade de alteração do planejamento cirúrgico para remoção do elemento ou até mesmo a sugestão de uma abordagem alternativa para reduzir 0 risco, como por exemplo, a coronectomia ${ }^{36}$. No entanto, para a maioria dos pacientes a indicação primária de tratamento é a mesma baseando-se em RP ou TCFC ${ }^{52}$. A questão é que observamos um aumento de confiança por parte do cirurgião dentista para definir o tratamento após a avaliação da TCFC ${ }^{45}$.

$O$ fato de existir na literatura indicativos que avaliam como superior a aquisição de imagem obtida pela TC sobre a RP para diagnóstico da real proximidade da raiz do terceiro molar com o canal mandibular, pode induzir os profissionais a prescreverem erroneamente uma dose excessiva de radiação para avaliar o risco de distúrbios sensoriais causados pela lesão do $\mathrm{NAI}^{48}$. As evidências atualizadas postulam que a TCFC não deve ser utilizada como método de rotina na remoção dos TMI, devendo ser aplicadas somente quando o cirurgião tem uma questão clínica muito específica que não pode ser respondida por imagens convencionais (panorâmica e / ou intraoral) $^{40,52}$.

CONCLUSÃO

A literatura aponta a interrupção da linha branca do canal, escurecimento da raiz, desvio do canal e seu estreitamento como sinais de alto risco que precisam ser levados em consideração no exame de radiografia panorâmica pré-operatória. Dessa forma o exame de eleição para a avaliação do risco continua sendo a radiografia panorâmica porque a tomografia computadorizada não parece reduzir as chances de lesão nervosa, possui dose maior de radiação e custo mais elevado. Os cirurgiões, entretanto, devem estar cientes das limitações dos marcadores radiográficos panorâmicos e devem considerar imagens mais detalhadas apenas em casos específicos.

AGRADECIMENTOS

O presente trabalho foi realizado com apoio da Fundação Universidade Federal de Mato Grosso do Sul - UFMS/MEC - Brasil. REFERÊNCIAS

1. Zandi $M$, Shokri A, Malekzadeh $H$, Amini $P$, Shafiey $P$. Evaluation of third molar development and its relation to chronological age: a panoramic radiographic study. Oral Maxillofac Surg. 2015;19:183-89.

2. Chou YH, Ho PS, Ho KY, Wang WC, Hu KF. Association between the eruption of the third molar and caries and periodontitis distal to the second molars in elderly patients. Kaohsiung $\mathrm{J}$ Med Sci. 2017;33(5):246-51.

3. L i ZB, Qu HL, Zhou LN, Tian BM, Chen FM. Influence of Non-Impacted Third Molars on Pathologies of Adjacent Second Molars: A Retrospective Study. J Periodontol. 2017;88(5):450-56.

4. Steed MB. The indications for third-molar extractions. J Am Dent Assoc. 2014;145(6): 570-73. 
5. Mortazavi H, Baharvand $M$. Jaw lesions associated with impacted tooth: A radiographic diagnostic guide. Imaging Sci Dent. 2016;46(3):147-57.

6. Hatami A, Dreyer C. The extraction of first, second or third permanent molar teeth and its effect on the dentofacial complex. Aust Dent J. 2019;64(4):302-11.

7. Peker I, Sarikir C, Alkurt MT, Zor ZF. Panoramic radiography and cone-beam computed tomography findings in preoperative examination of impacted mandibular third molars. BMC Oral Health. 2014;14(1):1-7.

8. Matzen LH, Schropp L, Spin-Neto R, Wenzel A. Radiographic signs of pathology determining removal of an impacted mandibular third molar assessed in a panoramic image or CBCT. Dentomaxillofac Radiol. 2017;46(1): 20160330.

9. Huang C-K, Lui M-T, Cheng D-H. Use of panoramic radiography to predict postsurgical sensory impairment following extraction of impacted mandibular third molars. J Chinese Med Assoc. 2015;78(10):617-22.

10.Zandi M, Shokri A, Heidari A, Peykar EM. Objectivity and reliability of panoramic radiographic signs of intimate relationship between impacted mandibular third molar and inferior alveolar nerve. Oral Maxillofac Surg. 2015;19:43-8.

11. Su N, Wijk A, Berkhout E, Sanderink G, Lange $\mathrm{J}$ De, Wang $\mathrm{H}$, et al. Predictive Value of Panoramic Radiography for Injury of Inferior Alveolar Nerve After Mandibular Third Molar Surgery. J Oral Maxillofac Surg. 2017;75(4):663-79.

12. Umar G, Obisesan O, Bryant C, Rood JP. Elimination of permanent injuries to the inferior alveolar nerve following surgical intervention of the "high risk" third molar. $\mathrm{Br} \mathrm{J}$ Oral Maxillofac Surg. 2013;51(4):353-57.

13. Suomalainen A, Ventä I, Mattila M, Turtola L, Vehmas T, Peltola JS. Reliability of CBCT and other radiographic methods in preoperative evaluation of lower third molars. Oral Surgery, Oral Med Oral Pathol Oral Radiol Endodontology. 2010;109(2):276-84.

14. Susarla SM, Sidhu HK, Avery LL, Dodson TB. Does Computed Tomographic Assessment of Inferior Alveolar Canal Cortical Integrity Predict Nerve Exposure During Third Molar Surgery? J Oral Maxillofac Surg. 2010;68(6):1296-303.

15. Grunheid T, Schieck JRK, Pliska BT, Ahmad M, Larson BE. Dosimetry of a cone-beam computed tomography machine compared with a digital $x$-ray machine in orthodontic imaging. Am J Orthod Dentofac Orthop. 2012;141(4):436-43.

16. Szalma J, Lempel E, Jeges S, Szabó G, Olasz $L$. The prognostic value of panoramic radiography of inferior alveolar nerve damage after mandibular third molar removal: retrospective study of 400 cases. Oral Surgery, Oral Med Oral Pathol Oral Radiol Endodontology. 2010;109(2):294-302.

17.Jaju PP, Jaju SP. Cone-beam computed tomography: Time to move from ALARA to ALADA. Imaging Sci Dent. 2015;45(4):263-65.

18. Rafetto LK. Managing Impacted Third Molars. Oral Maxillofac Surg Clin North Am. 2015; 27(3):363-71.

19. Synan W, Stein K. Management of Impacted Third Molars. Oral Maxillofac Surg Clin North Am. 2020;32(4):519-59.

20. Matzen LH, Wenzel A. Efficacy of CBCT for assessment of impacted mandibular third molars: a review - based on a hierarchical model of evidence. Dentomaxillofac Radiol. 2015;44(1):20140180.

21. Atieh MA. Diagnostic Accuracy of Panoramic Radiography in Determining Relationship Between Inferior Alveolar Nerve and Mandibular Third Molar. J Oral Maxillofac Surg. 2010;68(1):74-82.

22. Lobbers HT, Matthews F, Damerau G, Kruse AL, Obwegeser JA, Gratz KWA, et al. Anatomy of impacted lower third molars evaluated by computerized tomography is there an indication for 3-dimensional imaging? Oral Surg Oral Med Oral Pathol Oral Radiol Endod. 2011;111(5):547-50.

23. Rood JP. Degrees of injury to the inferior alveolar nerve sustained during the removal of impacted mandibular third molars by the lingual split technique. Br J Oral Surg. 1983;21:103-16.

24. Xu G, Yang C, Fan X-D, Yu C-Q, Cai X-Y, Wang $Y$, et al. Anatomic relationship between impacted third mandibular molar and the mandibular canal as the risk factor of inferior alveolar nerve injury. Br J Oral Maxillofac Surg. 2013;51(8):215-19.

25. Candotto V, Oberti L, Gabrione F, Scarano A, Rossi D, Romano M. Complication in third molar extractions. J Biol Regul Homeost Agents. 2019;33(3):169-72.

26. Rood JP, Shehab BAAN. The radiological prediction of inferior alveolar nerve injury during third molar surgery. Br J Oral Maxillofac Surg. 1990;28(1):20-5.

27. Winstanley KL, Otway LM, Thompson L, Brook $\mathrm{ZH}$, King $\mathrm{N}$, Koong $\mathrm{B}$, et al. Inferior alveolar nerve injury: Correlation between indicators of risk on panoramic radiographs and the incidence of tooth and mandibular canal contact on cone-beam computed tomography scans in a Western Australian population. J Investig Clin Dent. 2018;9(3):12323.

28. Sanmartí-Garcia G, Valmaseda-Castellón E, Gay-Escoda C. Does computed tomography prevent inferior alveolar nerve injuries caused by lower third molar removal? J Oral Maxillofac Surg. 2012;70(1):5-11. 
29. Dalili Z, Mahjoub P, Sigaroudi AK. Comparison between cone beam computed tomography and panoramic radiography in the assessment of the relationship between the mandibular canal and impacted class $C$ mandibular third molars. Dent Res J. 2011;8(4):203-10.

30. Hasani A, Moshtaghin FA, Roohi P, Rakhshan V. Diagnostic value of cone beam computed tomography and panoramic radiography in predicting mandibular nerve exposure during third molar surgery. Int J Oral Maxillofac Surg. 2017;46(2):230-35.

31. Shahidi S, Zamiri B, Bronoosh P. Comparison of panoramic radiography with cone beam CT in predicting the relationship of the mandibular third molar roots to the alveolar canal. Imaging Sci Dent. 2013;43:105-9.

32. Jung $\mathrm{Y}-\mathrm{H}$, Nah K-S, Cho B-H. Correlation of panoramic radiographs and cone beam computed tomography in the assessment of a superimposed relationship between the mandibular canal and impacted third molars. Imaging Sci Dent. 2012;42:121-27.

33. Neves FS, Souza TC, Almeida SM, Haiter-Neto F, Freitas DQ, Bóscolo FN. Correlation of panoramic radiography and cone beam CT findings in the assessment of the relationship between impacted mandibular third molars and the mandibular canal. Dentomaxillofacial Radiol. 2012;41(7):553-57.

34. Ueda M, Nakamori K, Shiratori K, Igarashi T, Sasaki T, Anbo N, et al. Clinical Significance of Computed Tomographic Assessment and Anatomic Features of the Inferior Alveolar Canal as Risk Factors for Injury of the Inferior Alveolar Nerve at Third Molar Surgery. J Oral Maxillofac Surg. 2012;70(3):514-20.

35. Kubota S, Imai T, Nakazawa M, Uzawa N. Risk stratification against inferior alveolar nerve injury after lower third molar extraction by scoring on cone-beam computed tomography image. Odontology. 2020;108(1):124-32.

36. Umar G, Bryant C, Obisesan O, Rood JP. Correlation of the radiological predictive factors of inferior alveolar nerve injury with cone beam computed tomography findings. Oral Surg. 2010;3(3):72-82.

37. Eyrich G, Seifert B, Matthews F, Matthiessen U, Heusser CK, Kruse AL, et al. 3-Dimensional Imaging for Lower Third Molars: Is There an Implication for Surgical Removal? J Oral Maxillofac Surg. 2011;69(7):1867-72.

38. Pippi R, Santoro M, D'Ambrosio F. Accuracy of cone-beam computed tomography in defining spatial relationships between third molar roots and inferior alveolar nerve. Eur $J$ Dent. 2016;10(4):454-58.

39. Wang D, Lin T, Wang Y, Sun C, Yang L, Jiang $\mathrm{H}$, et al. Radiographic features of anatomic relationship between impacted third molar and inferior alveolar canal on coronal CBCT images: risk factors for nerve injury after tooth extraction. Arch Med Sci. 2018;14(3):532-40.

40. Hasegawa T, Ri S, Shigeta T, Akashi M, Imai Y, Kakei $Y$, et al. Risk factors associated with inferior alveolar nerve injury after extraction of the mandibular third molar-a comparative study of preoperative images by panoramic radiography and computed tomography. Int $\mathrm{J}$ Oral Maxillofac Surg. 2013;42(7):843-51.

41. Selvi F, Dodson TB, Nattestad A, Robertson K, Tolstunov L. Factors that are associated with injury to the inferior alveolar nerve in high-risk patients after removal of third molars. Br J Oral Maxillofac Surg. 2013;51(8):868-73.

42. Shiratori K, Nakamori K, Ueda M, Sonoda $T$, Dehari $\mathrm{H}$. Assessment of the Shape of the Inferior Alveolar Canal as a Marker for Increased Risk of Injury to the Inferior Alveolar Nerve at Third Molar Surgery: A Prospective Study. J Oral Maxillofac Surg. 2013;71(12): 2012-19.

43. Nakamori K, Tomihara K, Noguchi M. Clinical significance of computed tomography assessment for third molar surgery. World $\mathrm{J}$ Radiol. 2014;6(7):417-23.

44. Tachinami $\mathrm{H}$, Tomihara $\mathrm{K}$, Fujiwara $\mathrm{K}$, Nakamori K, Noguchi M. Combined preoperative measurement of three inferior alveolar canal factors using computed tomography predicts the risk of inferior alveolar nerve injury during lower third molar extraction. Int J Oral Maxillofac Surg.2017;46(11):1479-83.

45. Szalma J, Vajta L, Lovász BV, Kiss C, Soós B, Lempel E. Identification of Specific Panoramic High-Risk Signs in Impacted Third Molar Cases in Which Cone Beam Computed Tomography Changes the Treatment Decision. J Oral Maxillofac Surg. 2020;78(7):1061-70.

46. Ali S Al, Jaber M. Correlation of panoramic high-risk markers with the cone beam CT findings in the preoperative assessment of the mandibular third molars. J Dent Sci. 2020; 15(1):75-83.

47. Patel PS, Shah JS, Dudhia BB, Butala PB, Jani $Y$ V, Macwan RS. Comparison of panoramic radiograph and cone beam computed tomography findings for impacted mandibular third molar root and inferior alveolar nerve canal relation. Indian J Dent Res. 2020;31(1):91-102.

48. Telles-Araújo $G$ de $T$, Peralta-Mamani $M$, Caminha RDG, Moraes-da-Silva A de F, Rubira $\mathrm{CMF}$, Honório HM, et al. CBCT does not reduce neurosensory disturbances after third molar removal compared to panoramic radiography: a systematic review and meta-analysis. Clin Oral Investig. 2020;24(3):1137-49.

49. Clé-Ovejero A, Sánchez-Torres A, Camps-Font O, Gay-Escoda C, Figueiredo R, ValmasedaCastellón E. Does 3-dimensional imaging of the third molar reduce the risk of experiencing inferior alveolar nerve injury owing to 
extraction?: A meta-analysis. J Am Dent Assoc. 2017;148(8):575-83.

50. Petersen LB, Vaeth $M$, Wenzel A. Neurosensoric disturbances after surgical removal of the mandibular third molar based on either panoramic imaging or cone beam CT scanning: A randomized controlled trial (RCT). Dentomaxillofac Radiol. 2016;45(2):20150224.

51. Ghaeminia H, Gerlach NL, Hoppenreijs TJ, Kicken M, Dings JP, Borstlap WA, et al. Clinical relevance of cone beam computed tomography in mandibular third molar removal: a multicentre, randomised, controlled trial. J Craniomaxillofac Surg. 2015;43(10):2158-67.

52. Matzen LH, Berkhout E. Cone beam CT imaging of the mandibular third molar: a position paper prepared by the European Academy of DentoMaxilloFacial Radiology (EADMFR). Dentomaxillofac Radiol. 2019;48(5):20190039.

\section{CONFLITO DE INTERESSES}

Os autores declaram não haver conflitos de interesse

\section{AUTOR PARA CORRESPONDÊNCIA}

\section{Ellen Cristina Gaetti Jardim}

FAODO - UFMS

79070-900 Campo Grande - MS, Brasil

e-mail: ellen.jardim@ufms.br 